\title{
Microfluidic droplet content detection using integrated capacitive sensors
}

\author{
Pelin Kubra Isgor, Merve Marcali, Mert Keser, Caglar Elbuken* \\ UNAM-National Nanotechnology Research Center, Institute of Materials Science and Nanotechnology, Bilkent University, 06800 Ankara, Turkey
}

\section{A R T I C L E I N F O}

\section{Article history:}

Received 12 August 2014

Received in revised form

18 December 2014

Accepted 7 January 2015

Available online 14 January 2015

\section{Keywords:}

Microdroplets

Capacitive sensor

Coplanar electrodes

Droplet content sensing

\begin{abstract}
A B S T R A C T
Microfluidic capacitive sensors have been used for detection of droplets, however they have been lacking the sensitivity required for detecting the content of droplets. In this study, we developed a scalable, portable, robust and high sensitivity capacitive microdroplet content detection system using coplanar electrodes with nanometer thick silicon dioxide $\left(\mathrm{SiO}_{2}\right)$ passivation layer and off-the-shelf capacitive sensors. The microfluidic chip we have designed provides easy and rapid modification of droplet content by mixing two aqueous liquids at any given ratio. The change in dielectric constant of the droplet content leads to the change in capacitive signal. The dielectric content of droplets was modified continuously while corresponding capacitance signal was measured. The resolution of the system was measured as 3 dielectric permittivity units. The results were verified using a semiconductor parameter analyzer. The application specific integrated circuit used in this work enables a portable, low-cost detection system and matches the performance of bench-top analyzers. Automated and precise measurement of dielectric content in droplets for biochemical assay monitoring is a major application of the presented system.
\end{abstract}

(c) 2015 Elsevier B.V. All rights reserved.

\section{Introduction}

Microdroplet based microfluidic systems are very handy platforms for applications that require experimentation on large libraries of samples. The fundamental requirements of most microdroplet systems are formation of monodisperse droplets, measuring the rate of droplet formation and detecting the analytical content of droplets. Optical counting of droplets and optical detection of droplet content are the most prevalent techniques in the literature [1,2]. Optical detection often requires bulky components and fluorescent labeling that increases the complexity and cost of the system [3]. Quenching of labels and sterical interference with molecular binding are other drawbacks of fluorescent labeling techniques [4]. Although, optical systems can detect droplets at very high rates (on the order of $\mathrm{kHz}$ ), these systems are not scalable [5]. On the other hand, electrical sensing techniques provide a scalable and label free alternative for droplet content detection, which allows multiple sensors in a small footprint at very low cost. Using off-the-shelf and low cost electrical components for high sensitivity droplet content detection can take

\footnotetext{
* Corresponding author. Tel.: +90 312 2903550; fax: +90 3122664365.

E-mail address: elbuken@unam.bilkent.edu.tr (C. Elbuken).
}

microdroplet-based microfluidic systems one step further, and turn them into programmable and easy-to-use platforms.

Electrical sensing for droplet based microfluidics was initially focused on droplet counting using either resistive or capacitive measurements. Some groups have measured the change in resistance due to the presence of droplets between the electrodes [6,7]. Luo et al. utilized an electrochemical detection technique to measure droplet size and its ionic content [8]. Due to the significant change in conductivity of the dispersed and continuous phase, very high sensitivity can be obtained in resistive droplet monitoring. However, direct contact between the droplet and the electrode surface should be avoided to minimize the risk of electrolysis and pinning of droplets on electrode surface which eventually leads to cross-contamination between droplets [9].

Capacitive detection of droplets is a label free and non-contact detection method [10]. Chen et al. and Ren et al. have demonstrated capacitive sensing of droplets using coplanar electrodes $[11,12]$. Using electro-wetting on dielectric (EWOD) systems, they have showed droplet detection and droplet volume metering. Other groups have demonstrated detection of droplets and their content by using the capacitive component of impedance signal $[13,14]$. However, these studies utilize custom-made electronics that require highly specialized expertise [10]. In addition, these systems can only detect the presence of the droplet and they are not sensitive enough to detect the content of the droplets. 
Being able to characterize the content of microdroplets electrically opens up new avenues and will eventually lead to impedance spectroscopy of droplets. The previous studies that utilize off-theshelf electronic components fall short in providing the sensitivity that is required for droplet content detection $[15,16]$. Thus, there is need for off-the-shelf detection systems that provide high sensitivity for droplet content detection. This paper is aimed to fill this gap by demonstrating capacitive droplet content detection using commercially available integrated sensors. The application specific integrated circuit used in this work enables a high sensitivity, portable, low-cost detection unit and matches the performance of bench-top LCR analyzers. To the best of our knowledge, this study provides the most sensitive capacitive detection signal, which is on the order of $\mathrm{fF}$, using off-the-shelf components. Utilizing these sensors, it is possible to detect biochemical activity in droplets by measuring the dielectric content change.

In this study, we have designed a Y-junction microfluidic device that allows rapid and easy modification of droplet content by mixing two liquids, ethanol and distilled (DI) water. High sensitivity label-free droplet content detection was achieved using coplanar electrodes coated with a nanometer thick $\mathrm{SiO}_{2}$ passivation layer, commercially available, low-cost capacitive sensors and a microprocessor. The system was characterized by modifying the dielectric content of the droplets on the run and measuring the corresponding capacitance signal. The results were verified by comparative measurements of our detection system and a semiconductor parameter analyzer. The capacitive signal was enhanced by minimizing the thickness of the passivation layer that separates the coplanar electrodes from the microchannel. This system provides label free capacitive droplet content measurements which enables dielectric spectroscopy in droplet based systems.

\section{Experimental}

In this study, we conducted experiments using a microfluidic device schematically shown in Fig. 1. Droplets were formed using a T-junction geometry $[17,18]$. In order to change the droplet content on the run, we utilized a Y-junction that feeds two different aqueous phases. At the downstream of the droplet generation section, a mixing region and a detection region were placed. Detection region consists of coplanar electrodes that were placed after the mixing region. These electrodes were passivated by $360 \mathrm{~nm} \mathrm{SiO} 2$ dielectric layer. A photograph of the fabricated device is shown in Fig. 2.

During experiments, droplets were formed at varying ethanol and DI water concentrations. Mixing region ensured that ethanol and DI water were properly mixed to obtain homogeneous droplets. The measured capacitance signal changes due to the stark contrast between dielectric permittivity of the continuous and dispersed phase when a droplet enters the sensing region.

It is important to passivate the electrodes in order to prevent cross-contamination between droplets and pinning of droplets onto the electrodes. For passivation of electrodes, there are fundamentally two fabrication options: spin-coating and thin film deposition. We have studied both methods in detail in order to obtain a thin, pin-hole free uniform passivation layer coating to enhance the capacitance signal. We have passivated electrodes by spin coating of polydimethylsiloxane (PDMS), and also by plasma enhanced chemical vapor deposition (PECVD) of $\mathrm{SiO}_{2}$. We showed that the thickness of the passivation layer can be decreased by adding toluene to the PDMS mixture. We studied the effect of spin speed on the thickness of the PDMS and toluene-thinned PDMS coated layer [18].

\subsection{Design and fabrication of the microfluidic device}

Microfluidic device fabrication consists of three steps: patterning of coplanar electrodes on a glass slide, coating of passivation layer onto the electrodes, fabrication of microchannels and bonding. Electrodes were patterned on a glass slide using photolithography and lift-off. First hexamethyldisiloxane (HMDS) was spun on the glass slide, then AZ5214 positive photoresist (MicroChemicals) was coated at $4000 \mathrm{rpm}$ for $40 \mathrm{~s}$. Electrodes were patterned using a transparency mask at $50 \mathrm{~mJ} / \mathrm{cm}^{2}$ dose UV-light $(365 \mathrm{~nm})$ (EVG620). Then, UV-exposed glass slides were developed followed by deposition of $20 \mathrm{~nm} \mathrm{Cr}$ and $200 \mathrm{~nm} \mathrm{Cu}$ using magnetron sputtering (Vaksis, NanoD-4S). Electrode fabrication was completed by lift-off process in acetone using an ultrasonic agitator. Electrode thickness was measured using an Atomic Force Microscope (AFM) (PSIA, XE-100E, Suwon) with intermittent noncontact mode in air under ambient temperature. A Tap190Al-G probe with a force constant of approximately $48 \mathrm{~N} / \mathrm{m}$ and resonance frequency of $190 \mathrm{kHz}$ was used. By using the analytical model in our previous study, electrode width and gap were determined as $200 \mu \mathrm{m}$ and $50 \mu \mathrm{m}$, respectively [19].

Microfabricated electrodes were passivated with either spin coating of PDMS, toluene thinned PDMS or thin film deposition of $\mathrm{SiO}_{2}$ using PECVD. For studying the effect of spin speed on the thickness of the coated layer, the PDMS mixture was spun on microscope slides for $5 \mathrm{~min}$ at spin rates varying from $1000 \mathrm{rpm}$ to $7000 \mathrm{rpm}$. In order to get the toluene thinned PDMS, PDMS mixture was mixed with toluene in 1:3 $(\mathrm{w} / \mathrm{w})$ ratio. Then, the prepared mixture was spun coated on microscope slides for $2 \mathrm{~min}$ at same spin rates. After spin coating, glass slides were baked at $110^{\circ} \mathrm{C}$ for $2 \mathrm{~h}$. Variation of passivation layer thickness with respect to spin rate is discussed in Section 3.2. $\mathrm{SiO}_{2}$ passivation layer was deposited using PECVD (Vaksis, CVD-Handy). Target pressure, RF power and heater were set as $1 \mathrm{Torr}, 10 \mathrm{~W}$, and $200^{\circ} \mathrm{C}$, respectively. The thickness of the passivation layer was adjusted by the deposition duration.

Thickness of the $\mathrm{SiO}_{2}$, PDMS and toluene-thinned PDMS passivation layers were measured using a Variable Angle Spectroscopic Ellipsometer (J.A. Woollam, V-VASE). Ellipsometer was used at an incidence angle of $65^{\circ}$. Cauchy dispersion function was used to determine passivation layer thicknesses. In order to fit experimental data, refractive indices of PDMS and toluene-thinned PDMS were taken as 1.42 , and refractive index of $\mathrm{SiO}_{2}$ was taken as 1.55 .

The microchannels were fabricated using standard soft lithography methods [20]. Molds were fabricated by patterning SU-8 photoresist on a 4 inch silicon wafer using photolithography. The channel height was measured as $100 \mu \mathrm{m}$ using Stylus Profilometer (KLA Tencor, P6 Surface Profiler).

For molding process, PDMS mixture was prepared by mixing silicone elastomer and curing agent (Dow Corning, Sylgard 184) in $10: 1(w / w)$ ratio. Following degassing of the mixture, PDMS was cross-linked at $100{ }^{\circ} \mathrm{C}$ for $4 \mathrm{~h}$. After curing, inlet/outlet holes were punched using a biopsy punch. Finally, microchannels were bonded to the glass slides with passivated coplanar electrodes using oxygen plasma. The bonding between PDMS microchannels and $\mathrm{SiO}_{2}$ passivation layer was completed using a plasma cleaner (Nanoplas, DSB6000) at $50 \mathrm{~W}, 30^{\circ} \mathrm{C}$ for $1 \mathrm{~min}$. In order to enhance the bonding, the devices were baked at $100^{\circ} \mathrm{C}$ for $12 \mathrm{~h}$ immediately after plasma activated bonding.

\subsection{Measurement setup}

Microdroplets were formed using the T-junction microchannel geometry and a pressure pump (Elveflow). Silicone oil with 50 mPas viscosity (Ultrakim) was used as the continuous phase. The dispersed phase was composed of ethanol and DI water. 


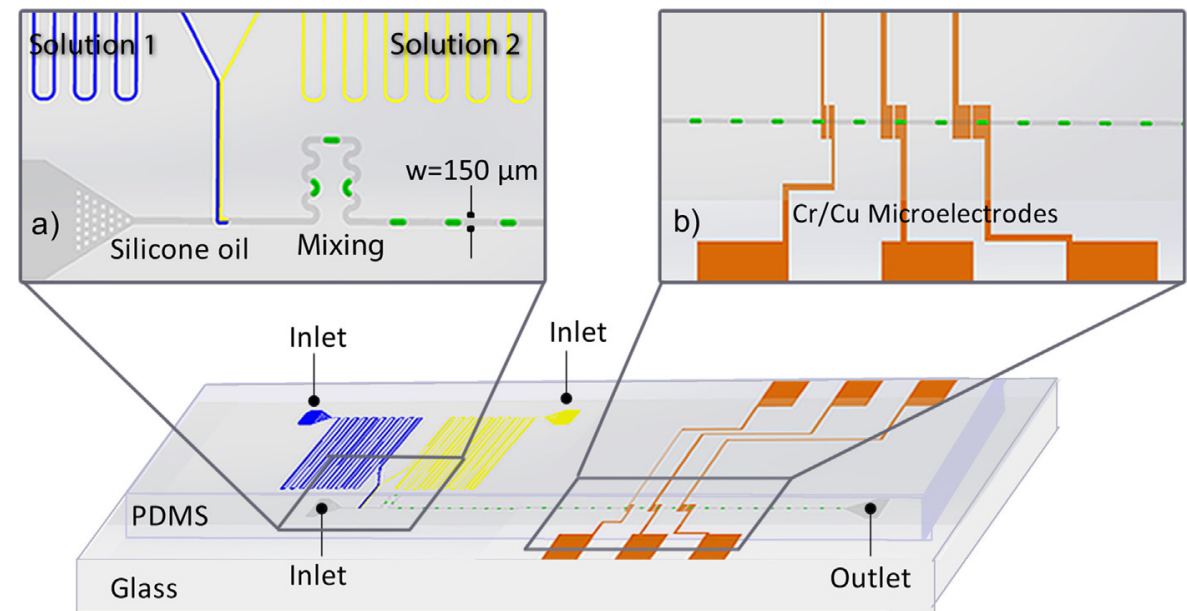

Fig. 1. Schematic of the microfluidic device. The insets show the (a) droplet generation region and mixing region, (b) detection region.

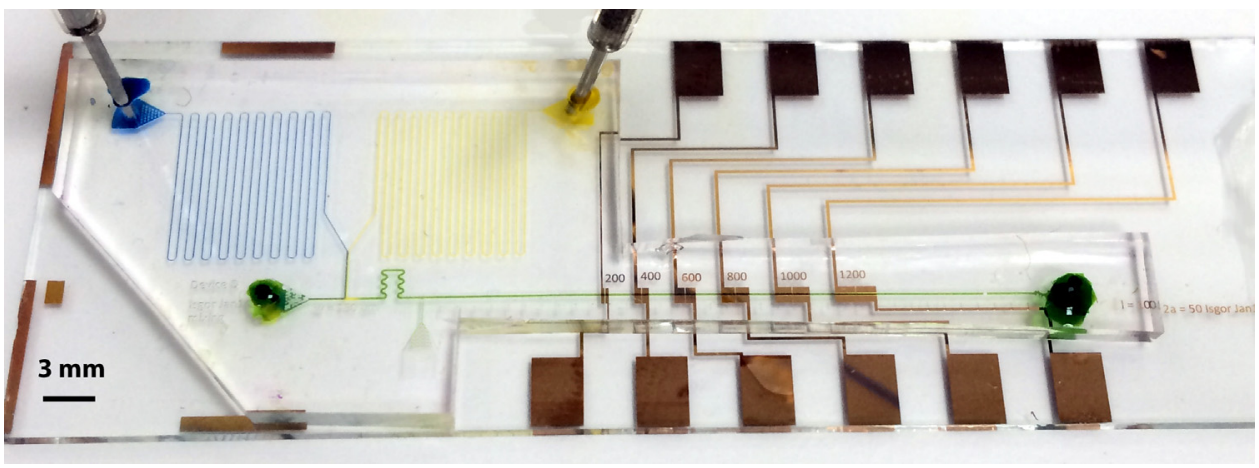

Fig. 2. Photograph of the fabricated microfluidic device. Channels were filled with dye solutions for clarity.

The dielectric permittivity contrast between continuous and dispersed phase is the basis of capacitive content detection of microdroplets. A cross-section of the detection region is shown in Fig. 3. The dielectric permittivity difference between silicone oil $\left(\varepsilon_{\mathrm{r}}=2.5\right)$ and microdroplet $\left(\varepsilon_{\mathrm{r}_{-} \text {ethanol }}=24<\varepsilon_{\mathrm{r}}<\varepsilon_{\mathrm{r}_{-} \text {water }}=80\right)$ causes an increase in the capacitance signal amplitude. The signal was measured using a capacitive-to-digital converter integrated circuit (Analog Devices, AD7746) which provides a low-cost, portable and scalable solution as opposed to bench-top measurement systems such as LCR analyzers. Full-scale linear capacitance measurement range of AD7746 is $\pm 4 \mathrm{pF}$ with a precision of $4 \mathrm{fF}$. AD7746 performs the measurement using an excitation signal at a constant frequency of $32 \mathrm{kHz}$. The excitation voltage can be digitally tuned by using the excitation set-up register. In order to prevent imprecision of the signal due to electrical wiring and keep the signal in

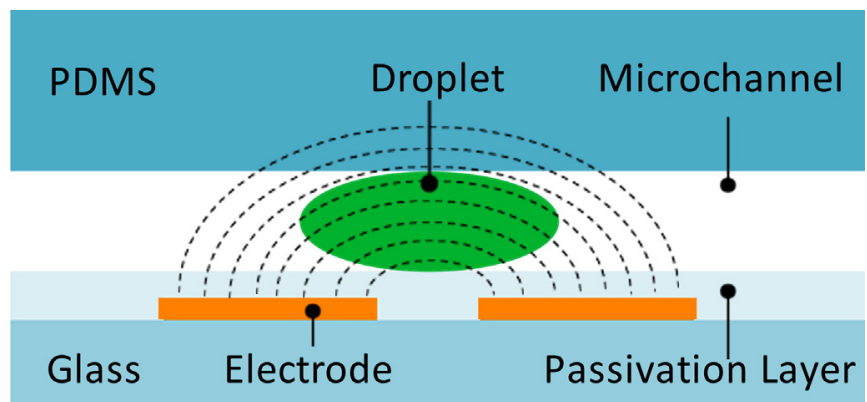

Fig. 3. Schematic of the cross-section of the detection region. the high-accuracy linear measurement range, internal offset capacitor was tuned for self-calibration during the test start-up cycle. AD7746 has two measurement modes but during this study, only single-ended mode was used. The read-out data rate was kept constant at $50 \mathrm{~Hz}$ to have enough sampling rate and low measurement noise. The discussion on selecting the read-out data rate can be seen in Supplementary Material. AD7746 was communicated with LabView using a USB-powered microcontroller (Arduino Duemilanove ATmega328). This microcontroller was used for displaying real-time capacitance signal through a LabView interface.

During the electrical measurements, droplets were observed using an inverted compound microscope in order to maintain the droplet size as similar as possible between different runs.

\subsection{Experimental procedure}

The device that is shown in Fig. 2 was used for the dielectric content measurement of droplets. Ethanol and DI water were separately driven from aqueous solution inlets using a pressure pump in order to change the dielectric content of the droplets. Blue dye was added to DI water in order to distinguish the separating boundary between DI water and ethanol (Fig. 4). During the measurements, the streamline separating the DI water (with blue dye) and ethanol was observed. The position of the separating boundary between DI water and ethanol is used to determine the mixing ratio. In addition, the blue color content of the droplets in the detection region was observed to form the aqueous droplets of predetermined concentrations.

For droplet content detection, coplanar electrodes that have a width of $200 \mu \mathrm{m}$ and a gap of $50 \mu \mathrm{m}$ were used. Experiments 
a)

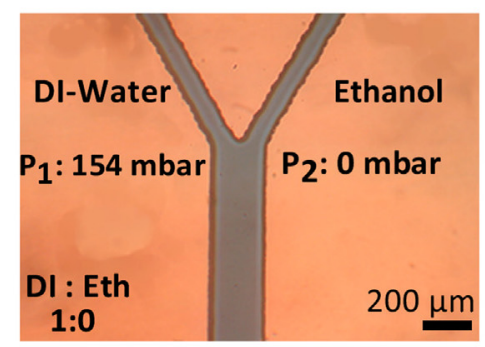

b)

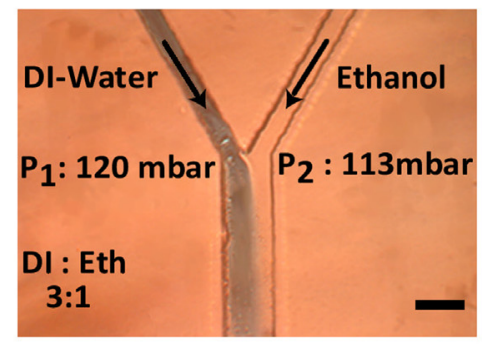

c)

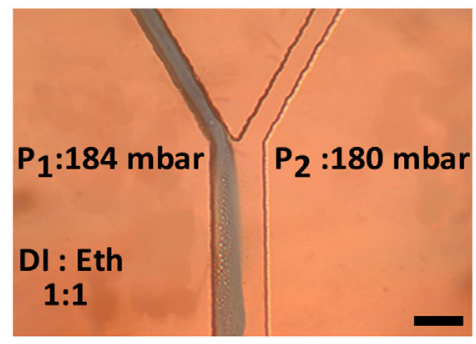

d)

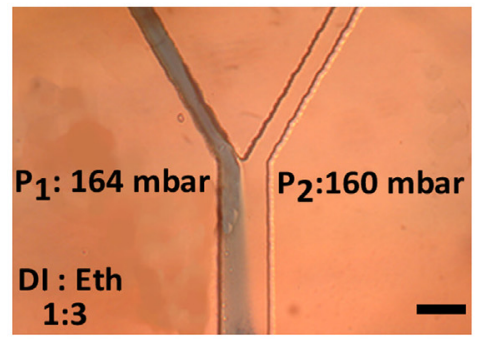

e)

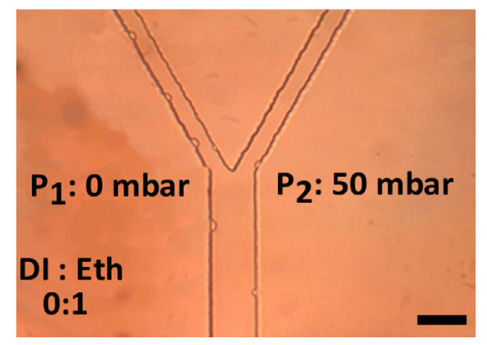

Microelectrode Sensor
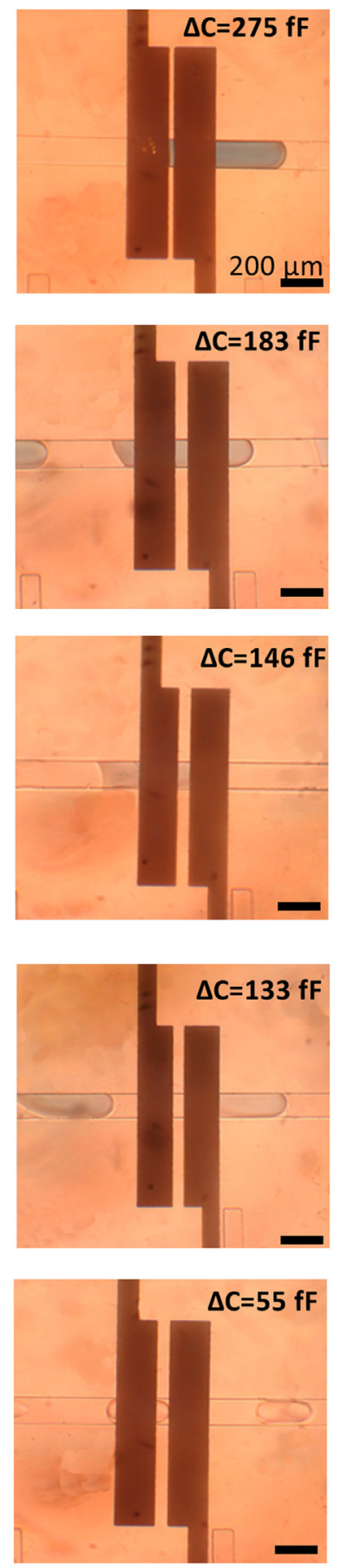

$\Delta C=$ Capacitance Signal (fF)
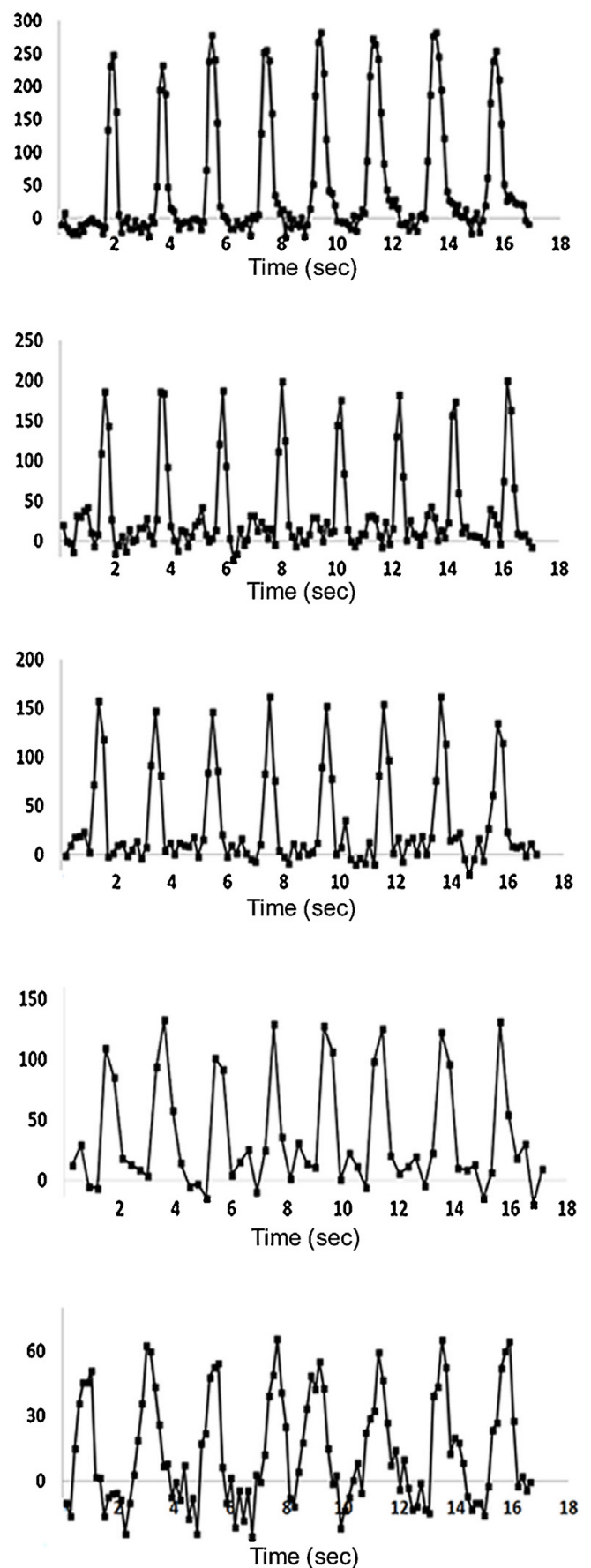

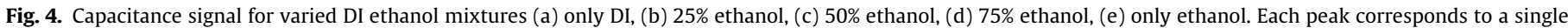
droplet in the sensing region. The plots show the data for $17 \mathrm{~s}$.

were performed at five different concentrations of ethanol DI water mixtures $(0 \%, 25 \%, 50 \%, 75 \%, 100 \%(v / v)$ ethanol concentration). The real-time capacitance signal was displayed through the LabView interface and recorded when droplet enters to the detection region. Then, signal amplitude for each droplet is measured from the recorded data. For each experiment snapshot images were captured at the Y-junction region and the detection region as shown in Fig. 4. The flow rates were fine tuned in order to have droplets of similar size and speed. In this study, plug-like droplets whose length is larger than the spacing between electrodes were formed. This ensured that droplets completely occupy the sensing domain.

For verification of the results and to determine the resolution of the system, seven different solutions of ethanol and DI water mixtures were prepared off the device with ethanol concentrations of $0 \%, 5 \%, 10 \%, 25 \%, 50 \%, 75 \%$ and $100 \%$ (v/v). These solutions were fed into one of the dispersed phase inlets while keeping the other inlet plugged. Droplets of these solutions were generated separately and capacitive droplet content measurements were performed as explained above. These results form the calibration plot 


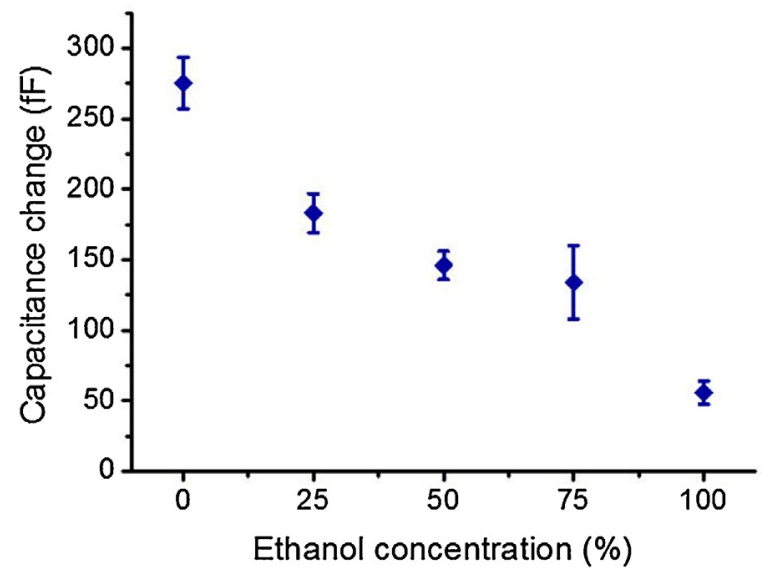

Fig. 5. Solution dependent capacitance signal amplitudes obtained from Fig. 4. Error bars denote one standard deviation across 50 droplet peaks.

that shows dependence of capacitance signal amplitude on ethanol concentration as shown in Fig. 6.

This calibration plot was also verified by recording capacitance signal amplitude using a high end bench-top semiconductor parameter analyzer (Keithley 4200). The same pre-mixed solutions were dispensed onto coplanar electrodes as large droplets $(50 \mu \mathrm{l})$ using a micropipette. The dispensed volume was high enough to completely fill the sensing domain of $200 \mu \mathrm{m}$ width and $50 \mu \mathrm{m}$ spacing electrodes. The results can be seen in Supplementary Material.

\section{Results and discussion}

\subsection{Droplet dielectric content measurement}

Fig. 4 summarizes the capacitive droplet content detection experiments. As seen in Fig. 4, DI droplets ( $0 \%$ ethanol) $\left(\varepsilon_{\mathrm{r}}=80\right)$ caused the highest capacitance change with an average of $275 \mathrm{fF}$, whereas ethanol droplets $(100 \%$ ethanol $)\left(\varepsilon_{\mathrm{r}}=24\right)$ caused the lowest capacitance change with an average of $55 \mathrm{fF}$. Decrease in DI water pressure caused a shift towards left at separating boundary at the Y-junction. Therefore, droplets with higher ethanol content led to smaller capacitance signal.

For the five cases demonstrated in Fig. 4, the capacitance signals were analyzed. Fig. 5 demonstrates the relationship between ethanol concentration of droplets and average capacitance signal amplitudes taken over 50 droplets. Increasing ethanol concentration causes a steady decrease in the detected signal.

Fig. 6 shows the calibration plot obtained by using pre-mixed solutions. This plot confirms that increase in ethanol concentration causes linear decrease in capacitive signal amplitude. DI ethanol mixtures were prepared in predetermined ratios with ethanol concentrations of $0 \%, 5 \%, 10 \%, 25 \%, 50 \%, 75 \%$ and $100 \%(v / v)$. For each ethanol concentration, capacitive signal amplitude was averaged over 50 droplets.

As seen from Fig. 5 and Fig. 6, the peak amplitude decreases with increasing ethanol concentration. The standard deviation is higher when mixing is performed on the run (Fig. 5) as opposed to pre-mixed solutions (Fig. 6). This is mainly due to the fact that the pressure fluctuations during droplet break-up process affect the upstream pressures and cause fluctuation in relative flow rates of ethanol and DI water. When the results in Fig. 5 are analyzed, it can be seen that the largest standard deviation is obtained for $75 \%$ ethanol concentration. It was observed that generating monodisperse droplets at this concentration is very challenging. As ethanol concentration of droplets was increased, the droplet formation at

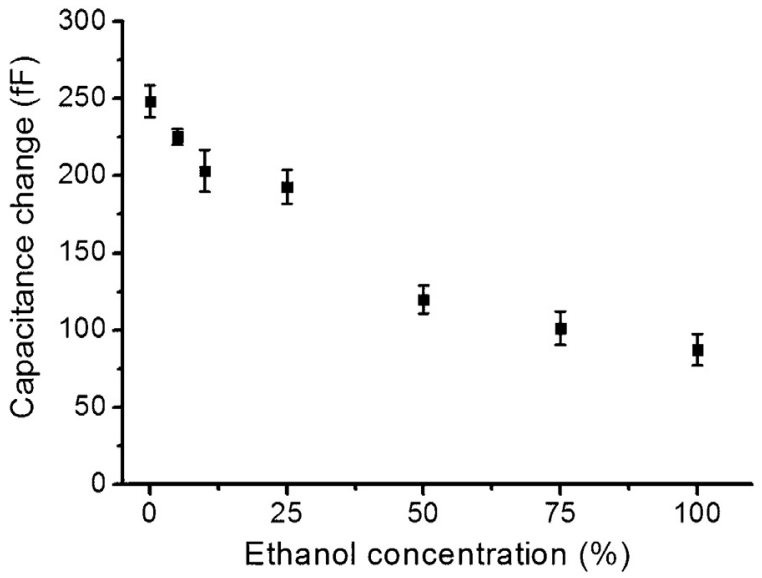

Fig. 6. Calibration plot obtained by pre-mixed solutions. Error bars denote one standard deviation across 50 droplet peaks.

the T-junction switched from squeezing regime to dripping regime (shown in Supplementary Material). In dripping regime, the droplet break-up point is a function of the ethanol and DI water ratio. During the formation of $75 \%$ ethanol concentration droplets, we have observed higher variation in the break-up point. This leads to higher variation in droplet size, causing the highest standard deviation in Fig. 5. It is also important to note that ethanol changes the surface properties of the PDMS microfluidic devices over time, which causes partial droplet wetting. After a few hours of continuous running, we have observed some droplet pinning on the side walls of channels as seen in Fig. 4d.

In a recent study, this sensor was used to measure droplet size and speed [19]. We have shown that any change in droplet size and speed affects the capacitance signal amplitude. Increasing droplet size or decreasing droplet speed leads to higher capacitance signal amplitude. Therefore, the variation in Fig. 5 and Fig. 6 is partially due to the variation in droplet size and speed. During all of the measurements, the pressure system was adjusted to form droplets of approximately $450 \mu \mathrm{m}$ in length. The monodispersity of droplets was measured as $5-10 \%$ from the recorded images of droplets. It was also observed that monodispersity was higher for the droplets that were generated using the pre-mixed solutions. This explains the smaller standard deviations observed in Fig. 6 compared to Fig. 5. Other than the measurements at $75 \%$ ethanol concentration, all the measurements follow a linear trendline in both measurements. It is also important to note that the $5 \%$ change in the droplet ethanol content can be resolved, which corresponds to a dielectric constant unit resolution of less than 3 .

The linear decrease of capacitance signal due to the increasing ethanol concentration was verified using a semiconductor parameter analyzer. During these verification measurements $50 \mu \mathrm{l}$ droplets were precisely pipetted onto the $375 \mathrm{~nm} \mathrm{SiO} 2$ passivated electrodes using the pre-mixed DI water ethanol solutions. The resultant plot can be found as Supplementary Material together with the photograph of the fabricated device. Since the sensing domain is completely covered with the solutions, the variation due to changing droplet size was eliminated. We observed a linear response with $R^{2}=0.99$. These measurements are in perfect agreement with the theoretical predictions that the capacitance signal amplitude linearly decreases with increasing ethanol concentrations. The signal amplitude for DI sample $\left(\varepsilon_{\mathrm{r}}=80\right)$ was $1054 \mathrm{fF}$, whereas it was $276 \mathrm{fF}$ for ethanol sample $\left(\varepsilon_{\mathrm{r}}=24\right)$. These results ensured that the variation and nonlinearity in the microfluidic system is due to the variation in droplet generation dynamics. The changing content of dispersed phase affects the viscosity as well as 


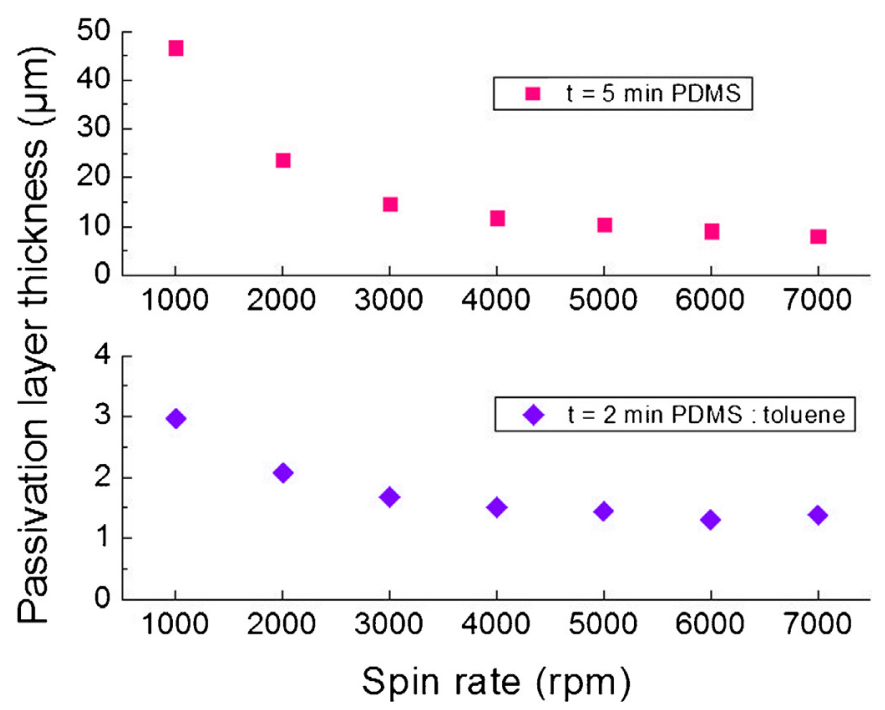

Fig. 7. PDMS and toluene thinned PDMS thickness. PDMS thickness (pink, square) for $5 \mathrm{~min}$. spinning time and PDMS:toluene 1:3 (w/w) thickness (blue, diamond) for $2 \mathrm{~min}$. spinning time (For interpretation of the color information in this figure legend, the reader is referred to the web version of the article.).

the wetting conditions, which in turn alters droplet formation at the T-junction.

In order to perform high sensitivity droplet content measurements, it is critical to minimize the baseline noise levels. Accuracy of AD7746 depends on the shielding of the system and electrodes as well as the measurement read-out rate. The read-out rate of AD7746 can be varied between $10 \mathrm{~Hz}$ and $90 \mathrm{~Hz}$. We have characterized the baseline noise level for DI water droplets measured at different data rates. The plots are given as Supplementary Material. We have observed that low data rates decrease baseline noise levels, however the sampling rate at these values $(10 \mathrm{~Hz})$ is not sufficient to sample the droplets. At $10 \mathrm{~Hz}$ sampling rate, droplets pass over the sensing region in approximately $500 \mathrm{~ms}$, which yields only four or five data points for each droplet. This leads to variation in the capacitance signal amplitude. Therefore, we have increased the data read-out rate of AD7746, until we have observed uniform signal for identical DI droplets. We have set our data rate as $50 \mathrm{~Hz}$. The baseline noise level at this speed was $15 \mathrm{fF}$. It is worth mentioning that the data rate for the sensor should be tailored depending on the flow rate of the droplets.

\subsection{Signal enhancement through thinner passivation layer}

For microfluidic systems that contain capacitive detection using coplanar electrodes, having a passivation layer is important to prevent cross-contamination between samples. Since the thickness of the passivation layer determines the distance of the microchannel from the electrodes (Fig. 3), it is critical to keep the thickness as low as possible to improve the signal amplitude. In order to compare different passivation layer types in terms of thickness, uniformity and quality, we used PDMS, toluene-thinned PDMS and $\mathrm{SiO}_{2}$. We have studied the effect of spin speed for PDMS and toluene-thinned PDMS to obtain thinner passivation layers. Thickness of PDMS depends on spin speed, duration, mixing ratio of silicone elastomer and curing agent, ambient conditions (temperature, humidity), time between PDMS preparation and spin coating. As it can be seen from Fig. 7, for a 5 min spinning time, increasing spin speed exponentially decreases PDMS passivation layer thickness.

It should be noted that in order to get consistent coating thicknesses for multiple devices, care should be taken for the duration between PDMS preparation and spin coating. Since PDMS gets gradually cured at room temperature, the more time passes between the preparation and spinning, the thicker the PDMS passivation layer becomes. Therefore, fresh PDMS mixture needs to be used in order to get thinner PDMS passivation layer.

Unlike PDMS, the thickness of toluene thinned PDMS does not decrease significantly for increasing spin speed, as seen in Fig. 7. It is because toluene thinned PDMS is much less viscous than PDMS. We have measured the viscosities of PDMS and 1:3 (w/w) toluene thinned PDMS at $25^{\circ} \mathrm{C}$ using a rheometer (Anton Paar, MCR-301) as $4220 \mathrm{mPa}$ s and $7.9 \mathrm{mPa}$ s, respectively.

Although spinner was used at its highest operation speed, both PDMS and toluene thinned PDMS thicknesses were higher than $1 \mu \mathrm{m}$. In order to further decrease the PDMS passivation layer thickness, spinning duration should be increased substantially. Still, the thickness of passivation layer will plateau at a certain thickness. Therefore, we used a thin film deposition technique, PECVD, in order to achieve uniformity and nanometer scale thickness.

We coated the coplanar electrodes with two different $\mathrm{SiO}_{2}$ passivation layer thicknesses and measured the capacitance signal amplitudes for DI droplets using the same Y-junction channel. For $180 \mathrm{~nm}$ thick $\mathrm{SiO}_{2}$ passivation layer, we achieved $514 \mathrm{fF}$ capacitance signal amplitude, whereas we got $275 \mathrm{fF}$ capacitance signal amplitude for $360 \mathrm{~nm}$ thick $\mathrm{SiO}_{2}$ passivation layer as shown in Fig. 4a. These results prove a 10 -fold capacitance signal amplitude enhancement for DI droplets as compared to our previous study where toluene-thinned PDMS passivation layer was used and the capacitance signal was measured as $26 \mathrm{fF}$ [19]. We have also confirmed the effect of passivation layer thickness on the signal level by measuring the capacitance change signal using PDMS coated electrodes. We can control the thickness of PDMS in a wide range as shown in Fig. 7. We have increased the thickness of PDMS passivation layer by successive spin coating steps in between each measurement. We have observed that the signal amplitude decreases significantly with increasing passivation layer thickness (Fig. S3 in Supplementary Material). Therefore, for applications requiring high sensitivity, it is important to minimize the thickness of the passivation layer, preferably using thin film deposition techniques to maximize the signal amplitude.

\subsection{Outlook}

As recently discussed in detail by Holger Becker, commercialization of lab-on-a-chip devices are facing several hurdles [21]. As the microfluidic community, it is an additional assignment for us to consider the manufacturability and scalability of the technologies we develop to overcome these hurdles. This study takes a step in that direction by utilizing very low cost electronics for high sensitivity measurement of droplet content (Arduino Nano \$20, AD7746 $\$ 4$ ). Droplet content detection is required for most of the droplet based biochemical assays. In addition to the droplet dielectric content measurement, this system can be used for very precise droplet size and speed detection as well as droplet counting. Monitoring metabolic activity of cells encapsulated in droplets using coplanar electrodes will be an interesting application of this system [5]. Another potential application of this system is dielectric indexing of droplets as an alternative to chemical indexing that is accomplished by encoded particles or labeled beads [22].

\section{Conclusions}

In this study, we have demonstrated high sensitivity capacitive droplet content detection using a scalable and cost-effective method. We showed that there is a linear relationship between 
the decrease in dielectric permittivity of droplet content and the capacitive signal. We verified these results by using a semiconductor parameter analyzer. In order to go beyond the routine uses of capacitive sensors as droplet detection sensors, it was required to increase the system sensitivity so that droplet content measurements were also possible. We have demonstrated that sensitivity can be improved by minimizing the thickness of the passivation layer. We showed that using commercially available, low-cost electrical components, droplet content can be detected in robust, precise and scalable manner at high sensitivity. The presented system is an alternative for bulky and costly bench-top analyzers.

\section{Acknowledgment}

The authors acknowledge support from European Union FP7 Marie Curie Career Integration Grant (no. 322019) and State Planning Organization of Turkey for the support of UNAM-National Nanotechnology Research Center.

\section{Appendix A. Supplementary data}

Supplementary data associated with this article can be found, in the online version, at http://dx.doi.org/10.1016/j.snb.2015.01.018.

\section{References}

[1] M.R. de Saint Vincent, S. Cassagnere, J. Plantard, J.P. Delville, Real-time droplet caliper for digital microfluidics, Microfluid. Nanofluid. 13 (2012) 261-271.

[2] Y.Zhao, Z.G. Xu, M. Parhizkar, J. Fang, X.G. Wang, T. Lin, Magnetic liquid marbles, their manipulation and application in optical probing, Microfluid. Nanofluid. 13 (2012) 555-564.

[3] J. Schemberg, A. Grodrian, R. Romer, G. Gastrock, K. Lemke, Online optical detection of food contaminants in microdroplets, Eng. Life Sci. 9 (2009) 391-397.

[4] U. Resch-Genger, M. Grabolle, S. Cavaliere-Jaricot, R. Nitschke, T. Nann, Quantum dots versus organic dyes as fluorescent labels, Nature Methods 5 (2008) $763-775$.

[5] H.N. Joensson, M. Uhlén, H.A. Svahn, Droplet size based separation by deterministic lateral displacement-separating droplets by cell-induced shrinking, Lab Chip 11 (2011) 1305-1310.

[6] N. Srivastava, M.A. Burns, Electronic drop sensing in microfluidic devices: automated operation of a nanoliter viscometer, Lab Chip 6 (2006) 744-751.

[7] M.C. Cole, P.J.A. Kenis, Multiplexed electrical sensor arrays in microfluidic networks, Sens. Actuators B-Chem. 136 (2009) 350-358.

[8] C.X. Luo, X.J. Yang, O. Fu, M.H. Sun, Q. Ouyang, Y. Chen, et al., Picoliter-volume aqueous droplets in oil: Electrochemical detection and yeast cell electroporation, Electrophoresis 27 (2006) 1977-1983.

[9] B.P. Cahill, R. Land, T. Nacke, M. Min, D. Beckmann, Contactless sensing of the conductivity of aqueous droplets in segmented flow, Sens. Actuators B-Chem. 159 (2011) 286-293.

[10] M. Demori, V. Ferrari, P. Poesio, D. Strazza, A microfluidic capacitance sensor for fluid discrimination and characterization, Sens. Actuators A-Phys. 172 (2011) 212-219.
[11] H. Ren, R.B. Fair, M.G. Pollack, Automated on-chip droplet dispensing with volume control by electro-wetting actuation and capacitance metering, Sens. Actuators B-Chem. 98 (2004) 319-327.

[12] J.Z. Chen, A.A. Darhuber, S.M. Troian, S. Wagner, Capacitive sensing of droplets for microfluidic devices based on thermocapillary actuation, Lab Chip 4 (2004) $473-480$.

[13] X.Z. Niu, M.Y.Zhang, S.L. Peng, W.J. Wen, P. Sheng, Real-time detection, control, and sorting of microfluidic droplets, Biomicrofluidics 1 (2007).

[14] E. Ghafar-Zadeh, M. Sawan, D. Therriault, A 0.18-mu m CMOS capacitive sensor lab-on-chip, Sens. Actuators A-Phys. 141 (2008) 454-462.

[15] M.F. Abdullah, P. Leow, M.A. Razak, F.C. Harun, On-chip droplets sensing using capacitive technique, J. Teknologi 61 (2013).

[16] J. Li, Y.X. Wang, E.K. Dong, H.S. Chen, USB-driven microfluidic chips on printed circuit boards, Lab Chip 14 (2014) 860-864

[17] T. Thorsen, R.W. Roberts, F.H. Arnold, S.R. Quake, Dynamic pattern formation in a vesicle-generating microfluidic device, Phys. Rev. Lett. 86 (2001) 4163

[18] P. Garstecki, M.J. Fuerstman, H.A. Stone, G.M. Whitesides, Formation of droplets and bubbles in a microfluidic T-junction - scaling and mechanism of break-up, Lab Chip 6 (2006) 437-446.

[19] C. Elbuken, T. Glawdel, D. Chan, C.L. Ren, Detection of microdroplet size and speed using capacitive sensors, Sens. Actuators A-Phys. 171 (2011) 55-62.

[20] D.C. Duffy, J.C. McDonald, O.J.A. Schueller, G.M. Whitesides, Rapid prototyping of microfluidic systems in poly(dimethylsiloxane), Anal. Chem. 70 (1998) 4974-4984.

[21] H. Becker, Chips, money, industry, education and the killer application, Lab Chip 9 (2009) 1659-1660.

[22] K. Braeckmans, S.C. De Smedt, M. Leblans, R. Pauwels, J. Demeester, Encoding microcarriers: present and future technologies, Nat. Rev. Drug Discov. 1 (2002) 447-456.

\section{Biographies}

Pelin Kubra Isgor received her B.Sc. degree in Electrical and Electronics Engineering from Koc University, Turkey in 2012. She pursues her M.Sc. degree in Department of Materials Science and Nanotechnology at National Nanotechnology Research Center-UNAM at Bilkent University, Ankara, Turkey. Her research interests include microdroplet-based microfluidic systems and lab-on-a-chip devices.

Merve Marcali received her B.Sc. degree in Biomedical Engineering from Baskent University, Turkey in 2013. In 2012, she worked as a summer researcher at Jade University of Applied Science in Germany and at Koc University. She pursues her M.Sc degree in Department of Materials Science and Nanotechnology at National Nanotechnology Research Center-UNAM at Bilkent University, Ankara, Turkey.

Mert Keser received his B.Sc. degree in Biomedical Engineering from Baskent University, Turkey in 2013. In 2012, he worked as a summer researcher at Koc University about microdroplet detection. During summer of 2013, he worked at National Nanotechnology Research Center-UNAM as a visiting researcher.

Caglar Elbuken received his B.Sc. degree in Electrical and Electronics Engineering from Bilkent University, Turkey in 2004 and his Ph.D. degree in Mechanical and Mechatronics Engineering from University of Waterloo, Canada in 2008. He worked as a postdoctoral associate at the Waterloo Microfluidics Laboratory for two years before joining to Abbott Point-of-Care as a senior R\&D scientist. Later, he joined Koc University as a research assistant professor. Since 2012, he is working at Bilkent University, National Nanotechnology Research Center as an assistant professor. His research interests include lab-on-a-chip devices, microdroplet-based microfluidic systems and sensing technologies for portable applications. 